\section{Increased Clostridium difficile Recurrences following Combined Proton Pump Inhibitor-Metronidazole Therapy}

To the Editor-The report by Hebert et al $^{1}$ provided strong support for increased risks of recurrent Clostridium difficile infections (CDI) in patients receiving either proton pump inhibitor (PPI) medications or primary metronidazole therapy. The authors did not, however, analyze $C$. difficile recurrence rates when both medications were administered concurrently. Recent studies support the possibility of inhibition of the effectiveness of metronidazole therapy by concurrent PPI administration. Al-Nassir et $\mathrm{al}^{2}$ randomized 52 patients with CDI to initially receive either vancomycin or metronidazole with a protocol-driven change to the alternative medication if initial therapy was unsatisfactory. Of 34 patients initially receiving metronidazole, 9 of the 10 who required change to vancomycin were PPI consumers, compared with only 10 of the 24 not requiring antibiotic alteration $(P<$ .02 ). Of 18 patients initially receiving vancomycin, 11 were PPI consumers, with only 1 requiring antibiotic alteration $(P<.01)$.

Musher et $\mathrm{al}^{3}$ reported 35 CDI patients for whom initial metronidazole therapy had failed; 27 of the 35 were PPI consumers. Twenty-six responded to nitazoxanide, suggesting less PPI interference with nitazoxanide therapy than with the prior metronidazole therapy.

Analysis by the authors of $C$. difficile recurrence rates among their patients who were concurrently receiving PPI and metronidazole therapy would assist practitioners in deciding whether or not these medications should be used in combination and whether or not vancomycin might be a preferred therapy for CDI in patients for whom concurrent PPI therapy should not be discontinued.

\section{ACKNOWLEDGMENTS}

This work was performed within my private practice of internal medicine. Potential conflicts of interest. The author reports no conflicts of interest relevant to this article. All authors submitted the ICMJE Form for Disclosure of Potential Conflicts of Interest, and the conflicts that the editors consider relevant to this article are disclosed here.

Harry W. Daniell, MD

Affiliation: 1. Department of Family Practice, University of California Medical School, Davis, California.

Address correspondence to Harry W. Daniell, MD, FACP, 2626 Edith Avenue, Suite A, Redding, CA 96001 (hwdaniell@aol.com).

Infect Control Hosp Epidemiol 2014;35(1):94-94
(C) 2013 by The Society for Healthcare Epidemiology of America. All rights reserved. 0899-823X/2014/3501-0018\$15.00. DOI: $10.1086 / 674400$

\section{REFERENCES}

1. Hebert C, Du H, Peterson LR, Robicsek A. Electronic health record-based detection of risk factors for Clostridium difficile infection relapse. Infect Control Hosp Epidemiol 2013;34:407-414.

2. Al-Nassir WN, Sethi AK, Nerandzic MM, Bobulsky GS, Jump RLP, Donskey CJ. Comparison of clinical and microbiological response to treatment of Clostridium difficile-associated disease with metronidazole and vancomycin. Clin Infect Dis 2008;47:5662 .

3. Musher DM, Logan N, Mehendiratta V, Melgarejo NA, Garud S, Hamill RJ. Clostridium difficile colitis that fails conventional metronidazole therapy: response to nitazoxanide. J Antimicrob Chemother 2007;59:705-710.

\section{Reply to Daniell}

To the Editor-We appreciate Dr Daniell's ${ }^{1}$ interesting comment on our article regarding electronic prediction of Clostridium difficile relapse. ${ }^{2}$

Dr Daniell cites two recent articles that suggest that the effect of proton pump inhibitors (PPI) on C. difficile primary treatment failure may be dependent on choice of initial treatment (metronidazole vs vancomycin or nitazoxanide). Our study was not designed to evaluate PPIs' effect on treatment failure, for two reasons:

1. Our focus was not on treatment failures. In fact, we avoided including patients with primary treatment failure by excluding any patient who received vancomycin or metronidazole in the follow-up period (after a normal course of treatment should have finished).

2. The variables that refer to metronidazole treatment and vancomycin treatment are not mutually exclusive. A patient could receive one, both, or neither of these medications. In fact, 190 (23\%) patients in our study received both medications at some point during the treatment period.

What our study was designed to address is Dr Daniell's question of whether relapse is more common among those who received a PPI and metronidazole, compared to those who received only metronidazole or those who received a PPI and vancomycin. Specifically, we found the following:

1. Of those subjects who received a PPI and metronidazole alone, the relapse rate was $31.5 \%(40 / 127)$, compared to $22.3 \%(78 / 349)$ of those patients who received metronidazole alone and no PPI. The unadjusted odds ratio (OR) 
was $1.60(95 \%$ confidence interval $[\mathrm{CI}], 1.02-2.51)$, $P=.04$.

2. Of those subjects who received a PPI and vancomycin alone, the relapse rate was $28.0 \%(7 / 25)$, compared to $10.3 \%(8 / 78)$ in those who received vancomycin alone and no PPI. The unadjusted OR was $3.40(95 \% \mathrm{CI}, 1.09$ 10.63), $P=.05$.

3. Of those who received any treatment (metronidazole or vancomycin or both) and a PPI, the relapse rate was $33.8 \%$ (68/201), compared to $21.7 \%$ (123/568) for those who did not receive a PPI. The unadjusted OR was $1.85(95 \% \mathrm{Cl}$, 1.30-2.64), $P<.01$.

4. Of those patients who received both metronidazole and vancomycin as well as a PPI, the relapse rate was $42.9 \%$ (21/49), compared to $26.2 \%(37 / 141)$ for those who received both agents but not a PPI. The unadjusted OR was 2.11 (95\% CI, 1.07-4.16), $P=.03$.

Our data suggest that PPI use is associated with a significantly increased risk of relapse, in unadjusted analyses, for both those who were treated with metronidazole and those who were treated with vancomycin. However, this analysis has several limitations. Our conclusions are based on unadjusted numbers, so unaccounted-for confounders may affect the results. Nevertheless, in our logistic regression model PPI use remained an independent predictor of relapse when adjusted for other factors, including age, metronidazole treatment, antibiotic exposure, and length of stay. In addition, the number of patients in our study who received vancomycin alone was small. In the subgroup of patients who received both metronidazole and vancomycin, there was an extremely high relapse rate in those who were given a PPI versus those who were not. It is possible that those patients had treatment failure with metronidazole; however, our data collection technique did not allow for that level of granularity.

In summary, our retrospective cohort study suggests that the use of a PPI is a predictor of readmission independent of choice of treatment. However, our study was not designed to address whether the concurrent use of PPI and metronidazole might lead to primary treatment failures. Dr Daniell presents an interesting insight that certainly deserves more study.

\section{ACKNOWLEDGEMENTS}

Financial support. The original study was supported by a grant from Merck to A.R. Merck has had no role in data analysis or the decision to publish this reply.

Potential conflicts of interest. All authors report no conflicts of interest relevant to this reply. All authors submitted the ICMJE Form for Disclosure of Potential Conflicts of Interest, and the conflicts that the editors consider relevant to this article are disclosed here.

\section{Courtney Hebert, $M{ }^{1}{ }^{1}$ Ari Robicsek, MD $^{2}$}

Affiliations: 1. Departments of Biomedical Informatics and Division of
Infectious Diseases, Ohio State University Wexner Medical Center, Columbus, Ohio; 2. Departments of Medicine and Health Information Technology, NorthShore University HealthSystem, Evanston, Illinois; and Pritzker School of Medicine, University of Chicago, Chicago, Illinois.

Address correspondence to Courtney Hebert, MD, Division of Infectious Diseases, Ohio State University Wexner Medical Center, 3190 Graves Hall, 333 West Tenth Avenue, Columbus, OH 43210 (courtney.hebert @osumc.edu).

Infect Control Hosp Epidemiol 2014;35(1):94-95

(C) 2013 by The Society for Healthcare Epidemiology of America. All rights reserved. 0899-823X/2014/3501-0019\$15.00. DOI: $10.1086 / 674401$

\section{REFERENCES}

1. Daniell HW. Increased Clostridium difficile recurrences following combined proton pump inhibitor-metronidazole therapy. Infect Control Hosp Epidemiol 2014;35:94 (in this issue).

2. Hebert C, Du H, Peterson LR, Robicsek A. Electronic health record-based detection of risk factors for Clostridium difficile infection relapse. Infect Control Hosp Epidemiol 2013;34:407-414.

\section{Identification of Clostridium difficile Ribotype 027 for the First Time in Mainland China}

To the Editor-Although several studies have shown that the incidence of infection with Clostridium difficile is increasing in Asia as a consequence of widespread use of broad-spectrum antibiotics, ${ }^{1}$ very little is known about the epidemiology of C. difficile-associated diarrhea in developing countries. The hypertoxigenic epidemic strain of $C$. difficile, named ribotype 027 , has also rarely been detected in Asia, especially in China. $^{2,3}$

In a retrospective study, we performed epidemiologic screening of patients with $C$. difficile infection (CDI) and investigated the characteristics and epidemiology of $C$. difficile-associated diarrhea in a large teaching hospital in South China. Fresh stool samples were collected from patients with suspected CDI over a 2-year period (December 2009-May 2012). Of the 3,660 stool samples, $572(15.6 \%)$ were positive for $C$. difficile toxin by direct polymerase chain reaction (PCR) ${ }^{4,5}$ Logistic regression analysis showed that previous antibiotic use $(P=.04)$ and CDI experience $(P<.01)$ were significant risk factors for toxin-positive diarrhea. $C$. difficile ribotype 027 was not found, although 7 cases of specimens detected binary toxin successfully.

We also studied the relationship between CDI and inflammatory bowel disease. Stool specimens from 406 patients with inflammatory bowel disease, including 241 patients with Crohn's disease and 165 with ulcerative colitis, were collected from January 2010 to April 2013 in Nanfang Hospital. Thirtyfour patients with Crohn's disease and 34 patients with ulcerative colitis were positive for $C$. difficile, with positive rates of $14.1 \%$ and $20.6 \%$, respectively. Three stool samples were 